\title{
Certain Grüss type inequalities involving the generalized fractional integral operator
}

\author{
Guotao Wang ${ }^{1}$, Praveen Agarwal ${ }^{2 *}$ and Mehar Chand ${ }^{3}$
}

${ }^{\text {*Correspondence: }}$

goyal.praveen2011@gmail.com

2Department of Mathematics, Anand International College of

Engineering, Jaipur, Rajasthan 303012, India

Full list of author information is available at the end of the article

\begin{abstract}
A remarkably large number of Grüss type fractional integral inequalities involving the special function have been investigated by many authors. Very recently, Kalla and Rao (Matematiche LXVI(1):57-64, 2011) gave two Grüss type inequalities involving the Saigo fractional integral operator. Using the same technique, in this paper, we establish certain new Grüss type fractional integral inequalities involving the Gauss hypergeometric function. Moreover, we also consider their relevances for other related known results.
\end{abstract}

MSC: 26D10; 26A33

Keywords: integral inequalities; Grüss inequality; fractional integrals and hypergeometric fractional integrals

\section{Introduction and preliminaries}

In recent years, a number of inequalities involving the fractional operators (like ErdélyiKober, Riemann-Liouville, Saigo fractional integral operators etc.) have been considered by many authors (see, e.g., [1-10]; for very recent work, see also [11] and [12]). The abovementioned works largely have motivated us to perform the present study.

We begin by recalling some known functions and earlier works.

Let $f$ and $g$ be two functions which are defined and integrable on $[a, b]$. Then the following inequalities hold (see also [7], [13, p.296]):

$$
l \leq f(x) \leq L, \quad m \leq g(x) \leq M
$$

Let, for each $x \in[a, b], l, L, m$ and, $M$ be real constants satisfying the inequalities (1.1). Then the following Grüss type inequalities hold:

$$
\begin{aligned}
& \left|\frac{1}{(b-a)} \int_{a}^{b} f(x) g(x) d x-\frac{1}{(b-a)} \int_{a}^{b} f(x) d x \cdot \frac{1}{(b-a)} \int_{a}^{b} g(x) d x\right| \\
& \quad \leq \frac{1}{4}(L-l)(M-m),
\end{aligned}
$$

where $\frac{1}{4}$ is a best possible constant.

The inequality (1.2) has various generalizations that have appeared in the literature, for example, e.g. $[5,6,13-16]$ and the references cited therein.

Very recently, Kalla and Rao [12] gave two Grüss type inequalities involving the Saigo fractional integral operator. Using the same technique, in this paper, we establish certain

\section{囪 Springer}

@2014 Wang et al.; licensee Springer. This is an Open Access article distributed under the terms of the Creative Commons Attribution License (http://creativecommons.org/licenses/by/2.0), which permits unrestricted use, distribution, and reproduction in any medium, provided the original work is properly cited. 
new Grüss type fractional integral inequalities involving the generalized fractional integral operator due to Curiel and Galué (see [17]). Moreover, we also consider their relevant connections with other known results.

Throughout the present paper, we shall investigate a fractional integral over the space $C_{\lambda}$ introduced in [18] and defined as follows.

Definition 1.1 The space of functions $C_{\lambda}, \lambda \in \mathbb{R}$, the set of real numbers, consists of all functions $f(x), x>0$, that can be represented in the form $f(x)=x^{p} f_{1}(x)$ with $p>\lambda$ and $f_{1} \in C[0, \infty)$, where $C[0, \infty)$ is the set of continuous functions in the interval $[0, \infty)$.

We define a fractional integral operator $K_{t}^{\alpha, \beta, \eta, \delta}$ associated with the Gauss hypergeometric function as follows.

Definition 1.2 Let $f \in C_{\lambda}$. For $\alpha>\max \{0,-(\delta+\eta+1)\}, \beta-1<\eta<0, \beta<1$ and $\delta>-1$ we define a fractional integral $K_{t}^{\alpha, \beta, \eta, \delta} f$ as follows:

$$
\left(K_{t}^{\alpha, \beta, \eta, \delta} f\right)(x)=\frac{\Gamma(1-\beta) \Gamma(\alpha+\delta+\eta+1)}{\Gamma(\eta-\beta+1) \Gamma(\delta+1)} x^{\beta+\delta}\left(I_{t}^{\alpha, \beta, \eta, \delta} f\right)(x)
$$

where $I_{t}^{\alpha, \beta, \eta, \delta}$ is the Gauss hypergeometric fractional integral of order $\alpha$ and is defined in the following.

Definition 1.3 Let $\alpha>0, \delta>-1, \beta, \eta \in \mathbb{R}$. Then the generalized fractional integral $I_{t}^{\alpha, \beta, \eta, \delta}$ (in terms of the Gauss hypergeometric function) of order $\alpha$ for real-valued continuous function $f(t)$ is defined by [17] (see also [19])

$$
\begin{aligned}
& I_{t}^{\alpha, \beta, \eta, \delta}\{f(x)\} \\
& \quad=\frac{x^{-\alpha-\beta-2 \delta}}{\Gamma(\alpha)} \int_{0}^{x} t^{\delta}(x-t)^{\alpha-1}{ }_{2} F_{1}\left(\alpha+\beta+\delta,-\eta ; \alpha ; 1-\frac{t}{x}\right) f(t) d t,
\end{aligned}
$$

where the function ${ }_{2} F_{1}(\cdot)$ appearing as a kernel for the operator (1.4) is the Gaussian hypergeometric function defined by

$$
{ }_{2} F_{1}(a, b ; c ; t)=\sum_{n=0}^{\infty} \frac{(a)_{n}(b)_{n}}{(c)_{n}} \frac{t^{n}}{n !}
$$

and $(a)_{n}$ is the Pochhammer symbol defined by $(n \in \mathbb{N})$, and

$$
(a)_{n}=a(a+1) \cdots(a+n-1) ; \quad(a)_{0}=1,
$$

where $\mathbb{N}$ denotes the set of positive integers.

The above integral (1.4) has the following commutative property:

$$
I_{t}^{\alpha, \beta, \eta, \delta} I_{t}^{a, b, c, d} f(x)=I_{t}^{a, b, c, d} I_{t}^{\alpha, \beta, \eta, \delta} f(x) .
$$


In the sequel, we use the following well-known result to establish our main results in the present paper:

$$
{ }_{2} F_{1}(a, b ; c ; 1)=\frac{\Gamma(c) \Gamma(c-a-b)}{\Gamma(c-a) \Gamma(c-b)} \quad\left(\Re(c-a-b)>0 ; c \in \Xi / \mathbb{Z}_{0}^{-}\right),
$$

where $\Xi$ and $/ \mathbb{Z}_{0}^{-}$denotes the sets of complex numbers and nonpositive integers, respectively.

Definition 1.4 Two functions $f$ and $g$ are said to be synchronous functions on $[0, \infty)$ if

$$
A(u, v)=(f(u)-f(v))(g(u)-g(v)) \geq 0 ; \quad u, v \in[0, \infty) .
$$

Next, we discuss some results regarding the fractional integral operator $K_{t}^{\alpha, \beta, \eta, \delta}$ which have been used in the present work.

Lemma 1.1 For $\mu>\max \{0,-(\eta-\beta)\}-1, \alpha>\max \{0,-(\delta+\eta+1)\}$; and $\eta-\beta>-1, \beta<1$, $\delta+\mu>-1$, we have

$$
K_{t}^{\alpha, \beta, \eta, \delta}\left(x^{\mu}\right)=\frac{\Gamma(1-\beta) \Gamma(\alpha+\delta+\eta+1) \Gamma(\delta+\mu+1) \Gamma(\mu-\beta+\eta+1)}{\Gamma(\eta-\beta+1) \Gamma(\delta+1) \Gamma(\mu-\beta+1) \Gamma(\mu+\delta+\alpha+\eta+1)} x^{\mu}
$$

and

$$
K_{t}^{\alpha, \beta, \eta, \delta}(C)=C,
$$

where $C$ is constant.

Proof Using the result (1.4), (1.3) reduces to

$$
\begin{aligned}
K_{t}^{\alpha, \beta, \eta, \delta}\left(x^{\mu}\right)= & \frac{\Gamma(1-\beta) \Gamma(\alpha+\delta+\eta+1) x^{\beta+\delta}}{\Gamma(\eta-\beta+1) \Gamma(\delta+1)} \frac{x^{-\alpha-\beta-2 \delta}}{\Gamma(\alpha)} \\
& \times \int_{0}^{x} t^{\delta+\mu}(x-t)^{\alpha-1}{ }_{2} F_{1}\left(\alpha+\beta+\delta,-\eta ; \alpha ; 1-\frac{t}{x}\right) d t .
\end{aligned}
$$

Using (1.5), (1.12) reduces to the following form:

$$
\begin{aligned}
K_{t}^{\alpha, \beta, \eta, \delta}\left(x^{\mu}\right)= & \frac{\Gamma(1-\beta) \Gamma(\alpha+\delta+\eta+1) x^{-\alpha-\delta}}{\Gamma(\eta-\beta+1) \Gamma(\delta+1)} \frac{1}{\Gamma(\alpha)} \\
& \times \sum_{n=0}^{\infty} \frac{(\alpha+\beta+\delta)_{n}(-\eta)_{n}}{(\alpha)_{n} n !} x^{-n} \int_{0}^{x} t^{\delta+\mu+1-1}(x-t)^{\alpha+n-1} d t \\
= & \frac{\Gamma(1-\beta) \Gamma(\alpha+\delta+\eta+1) x^{-\alpha-\delta}}{\Gamma(\eta-\beta+1) \Gamma(\delta+1)} \frac{1}{\Gamma(\alpha)} \sum_{n=0}^{\infty} \frac{(\alpha+\beta+\delta)_{n}(-\eta)_{n}}{(\alpha)_{n} n !} x^{-n} \\
& \times x^{\delta+\mu+\alpha+n+1-1} B(\delta+\mu+1, \alpha+n) \\
= & \frac{\Gamma(1-\beta) \Gamma(\alpha+\delta+\eta+1) \Gamma(\delta+\mu+1)}{\Gamma(\eta-\beta+1) \Gamma(\delta+1) \Gamma(\alpha+\delta+\mu+1)} \sum_{n=0}^{\infty} \frac{(\alpha+\beta+\delta)_{n}(-\eta)_{n}}{(\alpha+\delta+\mu+1)_{n} n !} x^{\mu}
\end{aligned}
$$




$$
\begin{aligned}
= & \frac{\Gamma(1-\beta) \Gamma(\alpha+\delta+\eta+1) \Gamma(\delta+\mu+1)}{\Gamma(\eta-\beta+1) \Gamma(\delta+1) \Gamma(\alpha+\delta+\mu+1)} \\
& \times{ }_{2} F_{1}(\alpha+\beta+\delta,-\eta ; \alpha+\delta+\mu+1 ; 1) x^{\mu} .
\end{aligned}
$$

Applying the result (1.8) to (1.13), after a little simplification, we easily arrive at the required result (1.10).

To prove (1.11), we again use the result (1.4), and (1.3) reduces to

$$
\begin{aligned}
K_{t}^{\alpha, \beta, \eta, \delta}(C)= & \frac{\Gamma(1-\beta) \Gamma(\alpha+\delta+\eta+1) x^{\beta+\delta}}{\Gamma(\eta-\beta+1) \Gamma(\delta+1)} \frac{x^{-\alpha-\beta-2 \delta}}{\Gamma(\alpha)} \\
& \times \int_{0}^{x} t^{\delta}(x-t)^{\alpha-1}{ }_{2} F_{1}\left(\alpha+\beta+\delta,-\eta ; \alpha ; 1-\frac{t}{x}\right) C d t .
\end{aligned}
$$

Using (1.5), (1.14) gets the following form:

$$
\begin{aligned}
K_{t}^{\alpha, \beta, \eta, \delta}(C)= & C \frac{\Gamma(1-\beta) \Gamma(\alpha+\delta+\eta+1) x^{-\alpha-\delta}}{\Gamma(\eta-\beta+1) \Gamma(\delta+1)} \frac{1}{\Gamma(\alpha)} \\
& \times \sum_{n=0}^{\infty} \frac{(\alpha+\beta+\delta)_{n}(-\eta)_{n}}{(\alpha)_{n} n !} x^{-n} \int_{0}^{x} t^{\delta+1-1}(x-t)^{\alpha+n-1} d t \\
= & C \frac{\Gamma(1-\beta) \Gamma(\alpha+\delta+\eta+1) x^{-\alpha-\delta}}{\Gamma(\eta-\beta+1) \Gamma(\delta+1)} \frac{1}{\Gamma(\alpha)} \sum_{n=0}^{\infty} \frac{(\alpha+\beta+\delta)_{n}(-\eta)_{n}}{(\alpha)_{n} n !} x^{-n} \\
& \times x^{\delta+\alpha+n+1-1} B(\delta+1, \alpha+n) \\
= & C \frac{\Gamma(1-\beta) \Gamma(\alpha+\delta+\eta+1)}{\Gamma(\eta-\beta+1) \Gamma(\alpha+\delta+1)} \sum_{n=0}^{\infty} \frac{(\alpha+\beta+\delta)_{n}(-\eta)_{n}}{(\alpha+\delta+1)_{n} n !} \\
= & C \frac{\Gamma(1-\beta) \Gamma(\alpha+\delta+\eta+1)}{\Gamma(\eta-\beta+1) \Gamma(\alpha+\delta+1)}{ }_{2} F_{1}(\alpha+\beta+\delta,-\eta ; \alpha+\delta+1 ; 1) .
\end{aligned}
$$

Using the result (1.8) in (1.15), after a little simplification, we easily arrive at the required result (1.11).

This completes the proof of the Lemma 1.1.

Lemma 1.2 Let $h \in C_{\lambda}$ and $m, M \in \mathbb{R}$ with $m \leq h(x) \leq M$. Then we have

$$
\begin{aligned}
& K_{t}^{\alpha, \beta, \eta, \delta} h^{2}(x)-\left(K_{x}^{\alpha, \beta, \eta, \delta} \operatorname{th}(x)\right)^{2} \\
& \quad=\left(M-K_{t}^{\alpha, \beta, \eta, \delta} h(x)\right)\left(K_{t}^{\alpha, \beta, \eta, \delta} h(x)-m\right)-K_{t}^{\alpha, \beta, \eta, \delta}(M-h(x))(h(x)-m),
\end{aligned}
$$

for all $x \in[0, \infty) ; \alpha>0, \delta>-1$, and $\beta, \eta \in \mathbb{R}$ with $\alpha+\beta+\delta \geq 0$ and $\eta \leq 0$.

Proof Let $h \in C_{\lambda}$ and $m, M \in R ; m \leq h(x) \leq M$, for all $x \in[0, \infty)$. Then, for any $u, v \in$ $[0, \infty)$, we have

$$
\begin{aligned}
& (M-h(u))(h(v)-m)+(M-h(v))(h(u)-m)-(M-h(u))(h(u)-m) \\
& -(M-h(v))(h(v)-m)=h^{2}(u)+h^{2}(v)-2 h(u) h(v) .
\end{aligned}
$$


If $h \in C_{\lambda}$, then $h$ is integrable on [0,x], $x>0$. Thus multiplying (1.17) by $\frac{u^{\delta}(x-u)^{\alpha-1}}{\Gamma(\alpha)}{ }_{2} F_{1}(\alpha+$ $\left.\delta+\beta,-\eta ; \alpha ; 1-\frac{u}{x}\right)$; using $u \in(0, x) ; x>0$ and integrating with respect to $u$ from 0 to $x$, and then applying Definition 1.2 and Lemma 1.1, we obtain

$$
\begin{aligned}
(M- & \left.K_{t}^{\alpha, \beta, \eta, \delta} h(x)\right)(h(v)-m)+(M-h(v))\left(K_{t}^{\alpha, \beta, \eta, \delta} h(x)-m\right) \\
& \quad-K_{t}^{\alpha, \beta, \eta, \delta}(M-h(x))(h(x)-m)-(M-h(v))(h(v)-m) \\
= & K_{t}^{\alpha, \beta, \eta, \delta} h^{2}(x)+h^{2}(v)-2 K_{t}^{\alpha, \beta, \eta, \delta} h(x) h(v) .
\end{aligned}
$$

Again multiplying (1.18) by

$$
\frac{v^{\delta}(x-v)^{\alpha-1}}{\Gamma(\alpha)}{ }_{2} F_{1}\left(\alpha+\delta+\beta,-\eta ; \alpha ; 1-\frac{v}{x}\right) ; \quad v \in(0, x) ; x>0
$$

then integrating with respect to $v$ from 0 to $x$, we obtain the required result (1.16). This completes the proof of Lemma 1.2.

\section{Main results}

In this section, we establish two inequalities involving the composition formula of the fractional integral (1.3) with a power function.

Theorem 2.1 Let $f$ and $g$ be two functions defined and integrable on $[a, b]$ with $f, g \in C_{\lambda}$ and satisfying the condition (1.1) on $[0, \infty)$. Thus we have

$$
\left|K_{t}^{\alpha, \beta, \eta, \delta} f g(x)-K_{t}^{\alpha, \beta, \eta, \delta} f(x) K_{t}^{\alpha, \beta, \eta, \delta} g(x)\right| \leq \frac{1}{4}(L-l)(M-m),
$$

for all $x \in[0, \infty) ; \alpha>0, \delta>-1$, and $\beta, \eta \in \mathbb{R}$ with $\alpha+\beta+\delta \geq 0$ and $\eta \leq 0$.

Proof Let us define a function

$$
A(u, v)=(f(u)-f(v))(g(u)-g(v)) \quad(u, v \in[0, x)) .
$$

First multiplying (2.2) by

$$
\begin{aligned}
& \frac{(u v)^{\delta}(x-u)^{\alpha-1}(x-v)^{\alpha-1}}{(\Gamma(\alpha))^{2}}{ }_{2} F_{1}\left(\alpha+\delta+\beta,-\eta ; \alpha ; 1-\frac{u}{x}\right) \\
& \times{ }_{2} F_{1}\left(\alpha+\delta+\beta,-\eta ; \alpha ; 1-\frac{v}{x}\right)
\end{aligned}
$$

and then integrating twice with respect to $u$ and $v$ from 0 to $x$, we obtain the following result with the aid of (1.3), (1.4), and property (1.5):

$$
\begin{aligned}
& \frac{1}{(\Gamma(\alpha))^{2}} \int_{0}^{x} \int_{0}^{x}(u v)^{\delta}(x-u)^{\alpha-1}(x-v)^{\alpha-1}{ }_{2} F_{1}\left(\alpha+\delta+\beta,-\eta ; \alpha ; 1-\frac{u}{x}\right) \\
& \quad \times{ }_{2} F_{1}\left(\alpha+\delta+\beta,-\eta ; \alpha ; 1-\frac{v}{x}\right) A(u, v) d u d v \\
& =2 K_{t}^{\alpha, \beta, \eta, \delta} f g(x)-2 K_{t}^{\alpha, \beta, \eta, \delta} f(x) K_{t}^{\alpha, \beta, \eta, \delta} g(x) .
\end{aligned}
$$


Making use of the well-known Cauchy-Schwarz inequality for a linear operator [13, Eq. (1.3), p.296], we find that

$$
\begin{aligned}
& \left(K_{t}^{\alpha, \beta, \eta, \delta} f g(x)-K_{t}^{\alpha, \beta, \eta, \delta} f(x) K_{t}^{\alpha, \beta, \eta, \delta} g(x)\right)^{2} \\
& \quad \leq\left(K_{t}^{\alpha, \beta, \eta, \delta} f^{2}(x)-\left(K_{t}^{\alpha, \beta, \eta, \delta} f(x)\right)^{2}\right)\left(K_{t}^{\alpha, \beta, \eta, \delta} g^{2}(x)-\left(K_{t}^{\alpha, \beta, \eta, \delta} g(x)\right)^{2}\right) .
\end{aligned}
$$

Since

$$
(L-f(x))(f(x)-l) \geq 0 \text { and }(M-g(x))(g(x)-m) \geq 0,
$$

we therefore have

$$
K_{t}^{\alpha, \beta, \eta, \delta}(L-f(x))(f(x)-l) \geq 0 \quad \text { and } \quad K_{t}^{\alpha, \beta, \eta, \delta}(M-g(x))(g(x)-m) \geq 0 .
$$

Thus by using Lemma 1.2, we have

$$
K_{t}^{\alpha, \beta, \eta, \delta} f^{2}(x)-\left(K_{t}^{\alpha, \beta, \eta, \delta} f(x)\right)^{2} \leq\left(L-K_{t}^{\alpha, \beta, \eta, \delta} f(x)\right)\left(K_{t}^{\alpha, \beta, \eta, \delta} f(x)-l\right)
$$

and

$$
K_{t}^{\alpha, \beta, \eta, \delta} g^{2}(x)-\left(K_{t}^{\alpha, \beta, \eta, \delta} g(x)\right)^{2} \leq\left(M-K_{t}^{\alpha, \beta, \eta, \delta} g(x)\right)\left(K_{t}^{\alpha, \beta, \eta, \delta} g(x)-m\right) .
$$

Using the inequalities (2.6) and (2.7), (2.4) reduces to the following form:

$$
\begin{aligned}
& \left(K_{t}^{\alpha, \beta, \eta, \delta} f g(x)-K_{t}^{\alpha, \beta, \eta, \delta} f(x) K_{t}^{\alpha, \beta, \eta, \delta} g(x)\right)^{2} \\
& \quad \leq\left(L-K_{t}^{\alpha, \beta, \eta, \delta} f(x)\right)\left(K_{t}^{\alpha, \beta, \eta, \delta} f(x)-l\right)\left(M-K_{t}^{\alpha, \beta, \eta, \delta} g(x)\right) \\
& \quad \times\left(K_{t}^{\alpha, \beta, \eta, \delta} g(x)-m\right) .
\end{aligned}
$$

Applying the well-known inequality $4 a b \leq(a+b)^{2}$; and using $a, b \in \mathbb{R}$ in the right-hand side of the inequality (2.8) and simplifying it, we obtain the required result (2.1). This completes the proof of Theorem 2.1.

Theorem 2.2 Let $f$ and $g$ be two synchronous functions on $[0, \infty)$. Then the following inequality holds:

$$
K_{t}^{\alpha, \beta, \eta, \delta} f g(x) \geq K_{t}^{\alpha, \beta, \eta, \delta} f(x) K_{t}^{\alpha, \beta, \eta, \delta} g(x),
$$

for all $x \in[0, \infty) ; \alpha>0, \delta>-1$, and $\beta, \eta \in \mathbb{R}$ with $\alpha+\beta+\delta \geq 0$ and $\eta \leq 0$.

Proof For the synchronous function $f$ and $g$, the inequality (1.9) holds for all $u, v \in[0, \infty)$.

This implies that

$$
f(u) g(u)-f(v) g(v) \geq f(u) g(v)+f(v) g(u) .
$$

Following the procedure of the Lemma 1.2 for applying the fractional integral $K_{t}^{\alpha, \beta, \eta, \delta}$, after a little simplification, we arrive at the required result (2.9). This completes the proof of Theorem 2.2. 


\section{Concluding remarks}

We consider some consequences of the results derived in the previous section. Following Curiel and Galué [17], the operator (1.4) would reduce immediately to the extensively investigated Saigo, Erdélyi-Kober, and Riemann-Liouville type fractional integral operators, respectively, given by the following relationships (see also [20] and [19]):

$$
\begin{aligned}
& I_{0, t}^{\alpha, \beta, \eta}\{f(t)\}=I_{t}^{\alpha, \beta, \eta, 0}\{f(t)\}=\frac{t^{-\alpha-\beta}}{\Gamma(\alpha)} \int_{0}^{t}(t-\tau)^{\alpha-1}{ }_{2} F_{1}\left(\alpha+\beta,-\eta ; \alpha ; 1-\frac{\tau}{t}\right) f(\tau) d \tau \\
& (\alpha>0 ; \beta, \eta \in \mathbb{R}), \\
& I^{\alpha, \eta}\{f(t)\}=I_{t}^{\alpha, 0, \eta, 0}\{f(t)\}=\frac{t^{-\alpha-\eta}}{\Gamma(\alpha)} \int_{0}^{t}(t-\tau)^{\alpha-1} \tau^{\eta} f(\tau) d \tau \quad(\alpha>0 ; \eta \in \mathbb{R})
\end{aligned}
$$

and

$$
R^{\alpha}\{f(t)\}=I_{t}^{\alpha,-\alpha, \eta, 0}\{f(t)\}=\frac{1}{\Gamma(\alpha)} \int_{0}^{t}(t-\tau)^{\alpha-1} f(\tau) d \tau \quad(\alpha>0) .
$$

Remarks We obtain the special cases of the operator $K_{t}^{\alpha, \beta, \eta, \delta}$ as follows by setting $\delta=0$, $\delta=\beta=0$ and $\delta=0$ and $\beta=-\alpha$ in (1.2). Immediately Definition 1.2 would reduce to the Saigo, Erdélyi-Kober, and Riemann-Liouville type fractional integral operators, respectively, given as follows:

$$
\begin{aligned}
& \left(K_{t}^{\alpha, \beta, \eta} f\right)(x)=\frac{\Gamma(1-\beta) \Gamma(\alpha+\eta+1)}{\Gamma(\eta-\beta+1)} x^{\beta}\left(I_{0}^{\alpha, \beta, \eta}, t f\right)(x), \\
& \left(K_{t}^{\alpha, \eta} f\right)(x)=\frac{\Gamma(\eta+\alpha+1)}{\Gamma(1+\eta)}\left(I^{\alpha, \eta} f\right)(x)
\end{aligned}
$$

and

$$
\left(K_{t}^{\alpha} f\right)(x)=\frac{\Gamma(\alpha+1)}{x^{\alpha}}\left(R^{\alpha} f\right)(x),
$$

where $\left(I_{0}^{\alpha, \beta, \eta}, t\right),\left(I^{\alpha, \eta}\right)$ and $\left(R^{\alpha}\right)$ are given by (3.1), (3.2), and (3.3), respectively.

We conclude our present investigation by remarking further that the results obtained here are useful in deriving various fractional integral inequalities involving such relatively more familiar fractional integral operators. For example, if we consider $\delta=0$ and make use of (3.1), Theorems 2.1 and 2.2 provide, respectively, the known fractional integral inequalities due to Kalla and Rao [12, pp.60-62, Eqs. (14) and (22)].

Again, for $\delta=0$ and $\beta=0$ in Theorems 2.1 and 2.2 and making use of the relation (3.2), Theorems 2.1 and 2.2 provide, respectively, the known fractional integral inequalities due to Kalla and Rao [12, p.62, Eqs. (24) and (25)].

Finally, taking $\delta=0$ and $\beta=-\alpha$ in Theorems 2.1 and 2.2 yields the known result due to Dahmani et al. [5, Theorem 3.1].

It is noted that the results derived in this paper are general in character and give some contributions to the theory of integral inequalities and fractional calculus. Moreover, they are expected to lead to some applications for establishing uniqueness of solutions in fractional boundary value problems, and in fractional partial differential equations. 


\section{Competing interests}

The authors declare that they have no competing interests.

\section{Authors' contributions}

All authors have equal contributions. All authors read and approved the final manuscript.

\section{Author details}

${ }^{1}$ School of Mathematics and Computer Science, Shanxi Normal University, Linfen, Shanxi 041004, China. ${ }^{2}$ Department of Mathematics, Anand International College of Engineering, Jaipur, Rajasthan 303012, India. ${ }^{3}$ Department of Mathematics, Fateh College for Women, Bathinda, 151103, India.

\section{Acknowledgements}

The authors are thankful to the worthy referees for their useful suggestions. This work is supported by the Natural Science Foundation for Young Scientists of Shanxi Province, China (No. 2012021002-3).

Received: 31 January 2014 Accepted: 25 March 2014 Published: 09 Apr 2014

\section{References}

1. Anastassiou, GA: Advances on Fractional Inequalities. Springer Briefs in Mathematics. Springer, New York (2011)

2. Belarbi, S, Dahmani, Z: On some new fractional integral inequalities. J. Inequal. Pure Appl. Math. 10(3), Article 86 (2009) (electronic)

3. Dahmani, Z: New inequalities in fractional integrals. Int. J. Nonlinear Sci. 9, 493-497 (2010)

4. Dahmani, Z, Mechouar, O, Brahami, S: Certain inequalities related to the Chebyshev's functional involving a type Riemann-Liouville operator. Bull. Math. Anal. Appl. 3(4), 38-44 (2011)

5. Dahmani, Z, Tabharit, L, Taf, S: New generalisations of Grüss inequality using Riemann-Liouville fractional integrals Bull. Math. Anal. Appl. 2(3), 93-99 (2010)

6. Dragomir, SS: Some integral inequalities of Grüss type. Indian J. Pure Appl. Math. 31(4), 397-415 (2002)

7. Grüss, D: Über das maximum des absoluten Betrages von $\frac{1}{(b-a)^{2}} \int_{a}^{b} f(x) d x \int_{a}^{b} g(x) d x$. Math. Z. 39, $215-226$ (1935)

8. Kuang, JC: Applied Inequalities. Shandong Sciences and Technology Press, Jinan (2004) (Chinese)

9. Mitrinovic, DS: Analytic Inequalities. Springer, Berlin (1970)

10. Sulaiman, WT: Some new fractional integral inequalities. J. Math. Anal. 2(2), 23-28 (2011)

11. Baleanu, D, Purohit, SD, Agarwal, P: On fractional integral inequalities involving hypergeometric operators. Chin. J. Math. 2014, Article ID 609476 (2014). doi:10.1155/2014/609476

12. Kalla, SL, Rao, A: On Grüss type inequalities for a hypergeometric fractional integral. Matematiche LXVI(1), 57-64 (2011)

13. Mitrinović, DS, Pečarić, JE, Fink, AM: Classical and New Inequalities in Analysis. Kluwer Academic, Dordrecht (1993)

14. Mercer, AMcD: An improvement of the Grüss inequality. J. Inequal. Pure Appl. Math. 6(4), 1-4 (2005)

15. Pachpatte, BG: On Grüss type integral inequalities. J. Inequal. Pure Appl. Math. 3(1), 1-5 (2002)

16. Pachpatte, BG: A note on Chebyshev-Grüss inequalities for differential equations. Tamsui Oxford Univ. J. Math. Sci. 22(1), 29-36 (2006)

17. Curiel, L, Galué, L: A generalization of the integral operators involving the Gauss' hypergeometric function. Rev. Téc. Fac. Ing., Univ. Zulia 19(1), 17-22 (1996)

18. Dimovski, IH: Operational calculus for a class of differential operators. C. R. Acad. Bulgare Sci. 19, 1111-1114 (1966)

19. Kiryakova, VS: Generalized Fractional Calculus and Applications. Pitman Res. Notes Math. Ser., vol. 301. Longman, Harlow (1994)

20. Saigo, M: A remark on integral operators involving the Gauss hypergeometric functions. Math. Rep. Kyushu Univ. 11, 135-143 (1978)

10.1186/1029-242X-2014-147

Cite this article as: Wang et al.: Certain Grüss type inequalities involving the generalized fractional integral operator. Journal of Inequalities and Applications 2014, 2014:147

\section{Submit your manuscript to a SpringerOpen ${ }^{\ominus}$ journal and benefit from:}

- Convenient online submission

- Rigorous peer review

- Immediate publication on acceptance

- Open access: articles freely available online

- High visibility within the field

- Retaining the copyright to your article 